\title{
Dielectric description of wakes and stopping powers in solids
}

\author{
Isabel Abril, ${ }^{1}$ Rafael Garcia-Molina, ${ }^{2}$ Cristian D. Denton, ${ }^{1}$ F. Javier Pérez-Pérez, ${ }^{3}$ and Néstor R. Arista ${ }^{4}$ \\ ${ }^{1}$ Departament de Física Aplicada, Universitat d'Alacant, Apartat 99, E-03080 Alacant, Spain \\ ${ }^{2}$ Departamento de Física, Universidad de Murcia, Apartado 4021, E-30080 Murcia, Spain \\ ${ }^{3}$ Institute of Polymer Technology and Materials Engineering/Mathematical Sciences, Loughborough University, Loughborough, \\ Leicestershire LE11 3TU, United Kingdom \\ ${ }^{4}$ Instituto Balseiro, Centro Atómico Bariloche, RA-8400 Bariloche, Argentina
}

(Received 31 July 1997)

\begin{abstract}
The dielectric description of the dynamical potential induced by swift protons in solids and the related stopping power is analyzed, using a combination of Mermin-type dielectric functions, which are fitted to available experimental data, to describe the optical properties of various materials. We apply this method to represent the energy loss functions of aluminum, silicon, amorphous carbon, and copper on a wide range of energy and momentum transfers. Using these functions we calculate the shape of the wake potential induced by swift protons; significant differences are obtained in the cases of carbon and copper, with respect to the results derived from simplified dielectric models. The energy loss functions are also applied to calculate the proton stopping power of each element, which are compared with experimental values. [S1050-2947(98)03007-8]
\end{abstract}

PACS number(s): 34.50.Bw, 77.22.- d, 61.46.+w

\section{INTRODUCTION}

The dielectric formulation has become one of the most used methods to describe the interaction of swift ions and other charged particles with matter. The use of this formalism to study the energy loss of charged particles was introduced by Fermi [1] in his classical treatment of the density effect in the stopping power of relativistic particles in dense media; since then, it has been a subject of continuous and growing interest. Subsequent developments made by Bohm and Pines [2], Lindhard [3], Hubbard [4], Nozières and Pines [5], Ritchie [6], among others, made it possible to extend the dielectric formulation (originally based on the atomicoscillator model of dispersive media) and to provide a more comprehensive description of quantum-mechanical effects in solids [7].

The dielectric function obtained by Lindhard [3] for a free-electron gas has been the basis of many applications in solid-state physics and particle-solid interaction phenomena. Other models have been developed to describe the dielectric response of semiconductors [8-11] using different approximations to represent the electron bands of these solids.

A large number of calculations of stopping powers of ions and electrons, and the inelastic mean free path of electrons in solids, have been carried out [12-14] using either the original Lindhard dielectric treatment, the semiconductor models, or other alternatives such as the Mermin dielectric function [15] or extensions of the Drude model [16-18]. In general, it seems that the dielectric formalism provides a reasonably good approximation to evaluate averaged quantities such as those mentioned above.

A recent analysis $[19,20]$ of experimental data, for aluminum and different allotropic forms of carbon, shows the possibilities and the limitations of various dielectric models when trying to represent the full frequency and wave-number dependence of the observed energy-loss function for these elements. In these works we proposed a representation of the energy loss function (ELF hereafter) using linear combina- tions of Mermin-type ELF. The characteristics of this model have been discussed before $[19,20]$; here we mention that it contains a minimum set of parameters (related to position, width, and intensity of the peaks in the energy loss function), which can be determined from experimental data, and describes with analytical functions the complete frequency and wave-number dependence of the ELF. The consistency with the exact $f$-sum rule of the dielectric formalism [7] is also built into the model.

As indicated above, some of the most important applications of the dielectric formulation deal with studies of dynamical interactions of swift ions and electrons with dense media. The way these interactions take place can be described in terms of the induced potential, giving the response of the medium to the perturbation created by the ion moving with velocity $v$. It has been known for some time [21-23] that this potential has the shape of a trailing wake (thereby the name wake potential), which follows the motion of the projectile with the same velocity $v$, so that it remains as a stationary perturbation of the medium as seen from the moving projectile's reference frame.

The importance of the wake potential in relation with various phenomena has been widely considered. In particular it becomes of interest in studying the dynamical interactions among correlated ions or ion clusters moving in solids [2429], as in molecular beam-foil experiments [30-32]: in studies of energy shifts, radiative transitions and mixing of bound states for ions moving within a solid [33-37], and in many experimental and theoretical studies of energy loss of ions in solids $[12,13]$.

The purpose of this paper is to develop a realistic representation of the wake potential, using a more accurate dielectric description adjusted for each particular solid according to the method described below. The model gives the ELF of each material for a wide range of frequencies and wave numbers, using a set of parameters determined from available experimental data, such as optical properties or electronenergy-loss spectroscopy [38-44]. 
Using this description we will study the general shape of the induced or wake potential created by protons moving in four solids of experimental interest: aluminum, silicon, amorphous carbon, and copper. In addition, we discuss the stopping power, which is a consequence of the retarding force acting on the projectile due to the self-induced electric field.

In the next section we briefly review the basic model used to describe the response of a free-electron gas to an external disturbing charge and the method used to represent in a close way the dielectric properties of real solids. This model will then be used in Sec. III to analyze the characteristics of the wake potential and to calculate, and compare with experimental data, the values of the proton stopping powers for the targets indicated before. The final conclusions are presented in Sec. IV.

\section{DIELECTRIC MODELS}

\section{A. Lindhard dielectric function}

The Lindhard [3] dielectric function, $\epsilon_{\mathrm{L}}(k, \omega)$, describes the response of a degenerate free-electron gas to an external (longitudinal) perturbation, in terms of the momentum transfer $\hbar k$ and energy transfer $\hbar \omega$. The function $\epsilon_{\mathrm{L}}(k, \omega)$ can be derived from the quantum perturbation theory [3] or following the random phase approximation (RPA) [7], and it is usually written in the form

$$
\epsilon_{\mathrm{L}}(k, \omega)=1+\frac{\chi^{2}}{z^{2}}\left[f_{1}(u, z)+i f_{2}(u, z)\right]
$$

using Lindhard's dimensionless variables, $u=\omega /\left(k v_{\mathrm{F}}\right)$ and $z=k /\left(2 k_{\mathrm{F}}\right)$, where $\chi^{2}=e^{2} /\left(\pi \hbar v_{\mathrm{F}}\right)$ is the density parameter, $v_{\mathrm{F}}$ is the Fermi velocity of the target valence electrons, and $k_{\mathrm{F}}=m_{\mathrm{e}} v_{\mathrm{F}} / \hbar$. Atomic units (where $m_{\mathrm{e}}=e=\hbar=1$ ) will be used hereafter. The functions $f_{1}(u, z)$ and $f_{2}(u, z)$, which are related to the real and imaginary parts of $\epsilon_{\mathrm{L}}$, are given by [3]

$$
\begin{gathered}
f_{1}(u, z)=\frac{1}{2}+\frac{1}{8 z}[g(z-u)+g(z+u)], \\
f_{2}(u, z)=\left\{\begin{array}{l}
\frac{\pi}{2} u, \quad z+u<1 \\
\frac{\pi}{8 z}\left[1-(z-u)^{2}\right], \quad|z-u|<1<z+u \\
0, \quad|z-u|>1,
\end{array}\right.
\end{gathered}
$$

where

$$
g(x)=\left(1-x^{2}\right) \ln \left|\frac{1+x}{1-x}\right| .
$$

As is well known, this model describes the two basic modes of energy absorption by the electrons of the system, namely, (i) single-particle excitations (also called electronhole pair excitations), and (ii) collective or plasmon excitations. The regions in the $\omega-k$ plane where each of these excitations are relevant are the following: Single-particle excitations appear in the band region given by $|u-z|<1$ (or in terms of $\omega$ and $k: k^{2} / 2-k v_{\mathrm{F}}<\omega<k^{2} / 2+k v_{\mathrm{F}}$ ), which determines the region where $\operatorname{Im}\left[\epsilon_{\mathrm{L}}(k, \omega)\right] \neq 0$. Plasmon excita-

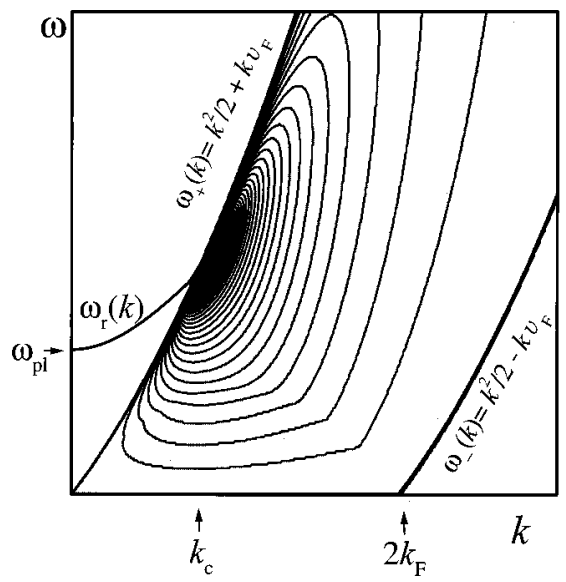

FIG. 1. Contour plot of the energy-loss function of a degenerate electron gas, $\operatorname{Im}\left(-1 / \epsilon_{\mathrm{L}}\right)$, as a function of the momentum $k$ and energy $\omega$ of the excitations. The line denoted by $\omega_{r}$ corresponds to the plasma resonance. The lines denoted by $\omega_{ \pm}=k^{2} / 2 \pm k v_{\mathrm{F}}$ delimit the single-particle excitations region. For the other symbols see the text.

tions arise from the resonance line where $\epsilon_{\mathrm{L}}(k, \omega)=0$; this determines a dispersion relation for the resonant frequency, $\omega_{r}(k)$, which gives the frequency of the longitudinal oscillations of the electron gas [7]. In the RPA picture these modes have an infinite lifetime, and a real frequency $\omega_{r}(k)$, through the range $0 \leqslant k \leqslant k_{c}$, where the value of $k_{c}$ corresponds to the point where the resonance line $\omega_{r}(k)$ intersects the upper boundary of the single-particle region [7], $\omega_{+}(k)=k^{2} / 2$ $+k v_{\mathrm{F}}$; the value of $\omega_{r}$ at $k=0$ is the plasmon frequency, $\omega_{\mathrm{pl}}$. In this range of $k$ values the absorption function corresponding to this resonance becomes a Dirac delta function, whereas for $k>k_{c}$ these modes can decay into electron-hole pairs, and therefore the shape of the resonance acquires a finite width. The regions of these excitations are illustrated in Fig. 1, where we show a plot of the Lindhard ELF, given by $\operatorname{Im}\left[-1 / \epsilon_{\mathrm{L}}(k, \omega)\right]$, as a function of $k$ and $\omega$.

\section{B. Mermin dielectric function}

One of the shortcomings of the Lindhard dielectric function [3] is the fact that it cannot represent the finite width of the plasma resonance in real materials (nor the finite plasmon lifetime associated with it). A straightforward attempt to introduce a relaxation-time approximation in the Lindhard dielectric function by turning the frequency $\omega$ into a complex frequency $\omega+i \gamma, \gamma$ being the damping rate of the plasmons, produces a conflict with the conservation of the local number of particles. This problem was first solved by Mermin [15], who derived an expression for the RPA dielectric function, in terms of the Lindhard dielectric function of complex frequency, but introducing in a consistent way a finite lifetime $\tau=1 / \gamma$ for the plasmons. The result for the Mermin dielectric function $\epsilon_{\mathrm{M}}(k, \omega)$ may be written in terms of $\epsilon_{\mathrm{L}}(k, \omega)$, by an appropriate combination of terms, as follows [15]:

$$
\epsilon_{\mathrm{M}}(k, \omega)=1+\frac{(1+i \gamma / \omega)\left[\epsilon_{\mathrm{L}}(k, \omega+i \gamma)-1\right]}{1+(i \gamma / \omega)\left[\epsilon_{\mathrm{L}}(k, \omega+i \gamma)-1\right] /\left[\epsilon_{\mathrm{L}}(k, 0)-1\right]}
$$

The properties of the ELF obtained from this representation will be illustrated in the next section. 


\section{Dielectric properties of real materials}

The analytical expressions given by the Lindhard [3] and Mermin [15] dielectric functions provide a convenient framework, based on which one can try to represent in a closer way the dielectric properties of real materials. In a few cases (like alkaline metals or aluminum) the Lindhard dielectric function already provides a reasonably good representation for the ELF of these elements. Here we will consider materials with more complicated electronic structure, which cannot be represented by a simple expression. However, the example of aluminum will be included in order to compare with a simple case where the Lindhard function may be used with some restrictions [20].

We construct the ELF of a material in the optical limit (i.e., at $k=0$ ) by a fit to the experimental ELF, which uses a linear combination of Mermin-type ELF

$$
\operatorname{Im}\left[\frac{-1}{\epsilon(k=0, \omega)}\right]_{\text {experim }}=\left\{\begin{array}{l}
\sum_{i} A_{i} \operatorname{Im}\left[\frac{-1}{\epsilon_{\mathrm{M}}\left(k=0, \omega ; \omega_{i}, \gamma_{i}\right)}\right] \quad \text { if } \omega<\omega_{i \text { edge }} \\
\sum_{i \text { shell }} A_{i \text { shell }} \operatorname{Im}\left[\frac{-1}{\epsilon_{\mathrm{M}}\left(k=0, \omega ; \omega_{i \text { shell }}, \gamma_{i \text { shell }}\right)}\right] \text { if } \omega \geqslant \omega_{i \text { edge }}
\end{array}\right.
$$

The first term in this equation $\left(\omega<\omega_{i \text { edge }}\right)$ represents the contribution to the excitation spectrum due to the outer electrons, with appropriate parameters $\omega_{i}, \gamma_{i}$, and $A_{i}$; the second term $\left(\omega \geqslant \omega_{i}\right.$ edge $)$ includes the participation of the electrons from the outermost atomic inner shell, which takes place when the excitation energy $\omega$ exceeds or is equal to the inner-shell edge energie $\omega_{i}$ edge . This model was previously applied to a few solids $[19,20]$. The values of $\left(\omega_{i}, \gamma_{i}, A_{i}\right)$ are related to the position, width, and height of each peak in the energy-loss spectrum, whereas the values of $\left(\omega_{i \text { shell }}, \gamma_{i \text { shell }}, A_{i \text { shell }}\right)$ are chosen to fit the shape of the ELF in the corresponding inner shells; all these parameters can be determined from optical data available at zero momentum transfer in a wide range of frequencies. The values of the coefficients $A_{i}$ and $A_{i}$ shell must satisfy the additional requirement that the frequency integral,

$$
N_{\text {eff }}(\omega)=\frac{1}{2 \pi^{2} n} \int_{0}^{\omega} d \omega^{\prime} \omega^{\prime} \operatorname{Im}\left[\frac{-1}{\epsilon\left(k, \omega^{\prime}\right)}\right]
$$

should be also in good agreement with the values derived from optical data for the effective number of electrons, $N_{\text {eff }}(\omega)$, that participate in the target excitations up to an energy $\omega$. In Eq. (7) $n$ is the atomic density of the target. When $\omega \rightarrow \infty, N_{\text {eff }}$ should tend to the total number of electrons per atom,

$$
N_{e}=\frac{1}{2 \pi^{2} n} \int_{0}^{\infty} d \omega^{\prime} \omega^{\prime} \operatorname{Im}\left[\frac{-1}{\epsilon\left(k, \omega^{\prime}\right)}\right],
$$

which is referred to as the $f$-sum rule.

It should be noted that the use of Mermin dielectric functions assures that the $f$-sum rule will be automatically satisfied for all values of the wave number $k$ if it is fulfilled at $k=0$. This is also an important advantage of this representation.

As the transfer energy increases, electrons from the atomic inner shells begin to participate in the excitation process. We have taken into account the contribution of these inner electrons to the energy-loss function by adding a new term for each inner shell [see Eq. (6)]; these new terms do not contribute to the ELF when the excitation energies $\omega$ are less than their corresponding inner-shell edge energies $\omega_{i}$ edge. The values of $\omega_{i}$ edge are obtained from Ref. [45], and the response of the inner electrons to external perturbations are derived from $\mathrm{x}$-ray scattering factors [46].

In order to guarantee that the partial sum rule given by Eq. (7) be verified for all values of $k$, we have written the evolution of $\omega_{i \text { edge }}$ with $k$ as follows

$$
\omega_{i \text { edge }}^{2}(k)=a_{i \text { edge }}+b_{i \text { edge }} k^{2}+c_{i \text { edge }} k^{4},
$$

where $a_{i}$ edge,$b_{i}$ edge and $c_{i}$ edge, are fitting constants for the edge of the $i$-shell of each material.

In Figs. 2(a)-2(d) we show the energy loss function, $\operatorname{Im}[-1 / \epsilon(k=0, \omega)]$, of aluminum, silicon, amorphous carbon, and copper. In each case, the dotted line represents the experimental data $[40,44,47]$, and the continuous line shows our fitted ELF, which was obtained by a sum of Mermin-type ELF, according to Eq. (6). The parameters used to fit the data shown for each material are given in Table I. As can be seen, aluminum is well described by a single Mermin-type ELF, whereas in the case of copper we have used five Mermintype ELF. The behavior of the ELF for aluminum for finite values of $k$ was analyzed in detail in Ref. [20] and a good general agreement with experimental data was found.

The case of silicon shows an intermediate behavior where a single Mermin-type ELF can still be used, although with a larger damping parameter than for aluminum. Carbon shows the interesting feature of a double plasma resonance (attributed to $\pi$ and $\pi+\sigma$ valence electrons [48]); to illustrate this behavior we found it convenient to describe its ELF by a sum of two Mermin-type ELF. A comparison of the dielectric properties and stopping powers of the various allotropic forms of carbon was given in Ref. [19]. Finally, copper shows a very complex structure in the absorption spectrum, related to interband transitions (cf. Ref. [40]), which is typical of the transition metals. It is worth noting that for the materials with a single peak in the ELF, like aluminum and silicon, the value of $\omega_{1}$ in Table I is given practically by the corresponding plasmon energy $\omega_{\mathrm{pl}}$.

In the projectile velocity range we will discuss in this paper not all the target electrons participate in the excitation 

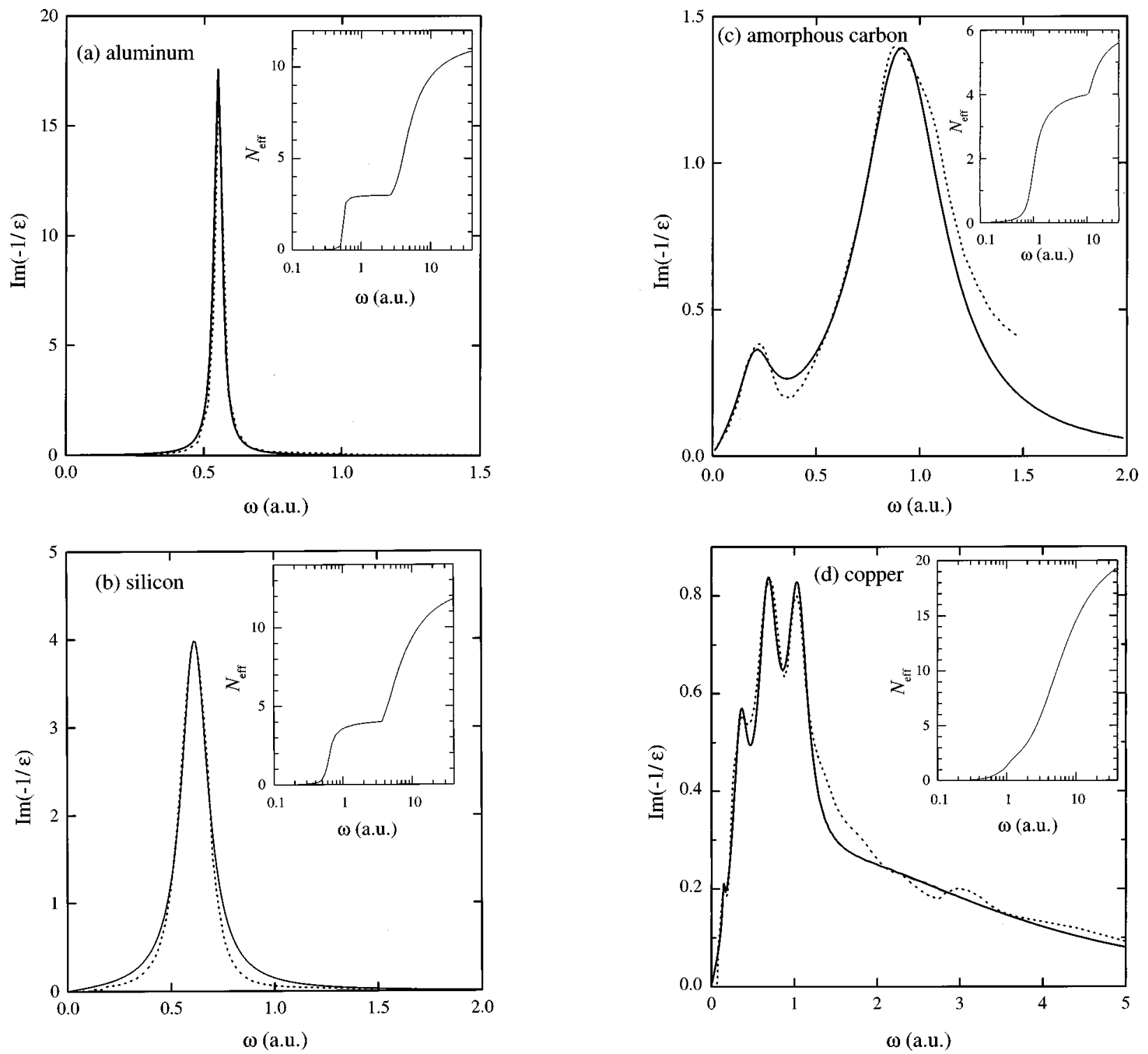

FIG. 2. Comparison of our fitted ELF (solid line) with the experimental ELF (dotted line) at $k=0$ : (a) aluminum [40], (b) silicon [44], (c) amorphous carbon [47], and (d) copper [40]. The insets show the effective number of electrons, $N_{\text {eff }}$, that participate in electronic excitations up to each energy $\omega$, calculated according to Eq. (7).

TABLE I. Parameters used to fit, through Eq. (6), the contribution of the outer electrons to the ELF of aluminum, silicon, amorphous carbon, and copper.

\begin{tabular}{lcccc}
\hline \hline \multicolumn{1}{c}{ Target } & $i$ & $\omega_{i}$ (a.u.) & $\gamma_{i}$ (a.u.) & \multicolumn{1}{c}{$A_{i}$} \\
\hline Aluminum & 1 & 0.551 & 0.035 & 1.1178 \\
Silicon & 1 & 0.620 & 0.156 & 0.9922 \\
Amorphous carbon & 1 & 0.230 & 0.21 & 0.2362 \\
& 2 & 0.945 & 0.49 & 0.7088 \\
Copper & 1 & 0.15 & 0.04 & 0.02 \\
& 2 & 0.37 & 0.22 & 0.2184 \\
& 3 & 0.70 & 0.30 & 0.2449 \\
& 4 & 1.05 & 0.30 & 0.1524 \\
& 5 & 2.90 & 5.6 & 0.3564 \\
\hline \hline
\end{tabular}

processes. Besides the valence electrons, we need to include the $K$ electrons for amorphous carbon, the $L$ electrons for aluminum and silicon, and the $M$ electrons for copper (but, due to the small value of $\omega_{M}$ edge, the contribution of these latter is not separated from that due to the valence electrons). In Table II we present the set of parameters used to account for the contribution of the above inner-shell electrons to the energy-loss processes.

The insets in Figs. 2(a)-2(d) show the effective number of electrons that participate in the electronic excitations of the target, given by Eq. (7); it can be appreciated that $N_{\text {eff }}$ displays correctly the behavior previously described, saturating at $\omega \simeq \omega_{i}$ edge to the number of electrons out of the $i$ shell, and jumping abruptly at $\omega>\omega_{i}$ edge, when new electrons enter into the excitations.

In order to illustrate the full frequency and wave-number dependence arising from this model we depict in Fig. 3 the ELF of (a) amorphous carbon and (b) copper, according to the present representation. By comparison with Fig. 1 we 
TABLE II. Parameters used to fit, according to Eqs. (6) and (9), the contribution of the inner electrons to the ELF of aluminum, silicon, amorphous carbon, and copper. Note that $\sqrt{a_{i} \text { edge }}$ $=\omega_{i}$ edge $(k=0)$, i.e., it corresponds to the threshold energy at which the electrons of the $i$ shell begin to participate in the excitations at $k=0$.

\begin{tabular}{lccccccc}
\hline \hline \multicolumn{1}{c}{ Target } & $i$ & $\begin{array}{c}\sqrt{a_{i \text { edge }}} \\
(\text { a.u. }\end{array}$ & $\begin{array}{c}b_{i} \text { edge } \\
(\text { a.u. })\end{array}$ & $\begin{array}{c}c_{i} \text { edge } \\
(\text { a.u. })\end{array}$ & $\begin{array}{c}\omega_{i} \text { shell } \\
(\text { a.u. })\end{array}$ & $\begin{array}{c}\gamma_{i \text { shell }} \\
(\text { a.u. })\end{array}$ & $A_{i \text { shell }}$ \\
\hline Aluminum & $L$ & 2.664 & 1.883 & 0.359 & 3.9 & 3.0 & 0.0666 \\
Silicon & $L$ & 3.668 & 1.182 & 0.340 & 4.2 & 4.9 & 0.05378 \\
$\begin{array}{c}\text { Amorphous } \\
\text { carbon }\end{array}$ & $K$ & 10.45 & 2.543 & 0.34 & 10.5 & 7.9 & 0.004078 \\
\hline \hline
\end{tabular}

observe important differences with respect to the freeelectron gas picture. It also can be appreciated that the peaked structures that appear at $k=0$ get smooth and finally disappear at larger wave numbers; this behavior, predicted by the Mermin-type ELF, coincides with the available experimental determinations of the ELF at $k \neq 0[49,50]$, and it is not well reproduced by other commonly used ELF models [17].

\section{INDUCED POTENTIAL AND STOPPING POWER}

Our purpose in this section is to make use of the energyloss functions already determined for the set of elements considered, and to analyze the sensitivity of the quantities of interest (wake potential and stopping power) with respect to the use of different dielectric models. Our calculations are based on the dielectric formalism, which is a linear response model, therefore some nonlinear corrections could be expected at low projectile velocities [13].

We will restrict ourselves here to the perturbation induced by a moving proton, and neglect charge exchange processes. This approach is justified for intermediate or large velocities, whereas for low velocities, or in the case of other ions, corrections due to effective-charge effects should be considered [51].

Following the dielectric formalism [23], we calculate the induced potential (or wake potential) produced by a proton moving with velocity $\vec{v}$ through a material characterized by its dielectric properties $\epsilon(k, \omega)$, from the expression

$$
\phi_{\text {ind }}(\vec{r})=\frac{Z}{2 \pi^{2}} \int \frac{d^{3} k}{k^{2}} e^{i \vec{k} \cdot \vec{r}}\left[\frac{1}{\epsilon(k, \vec{k} \vec{v})}-1\right],
$$
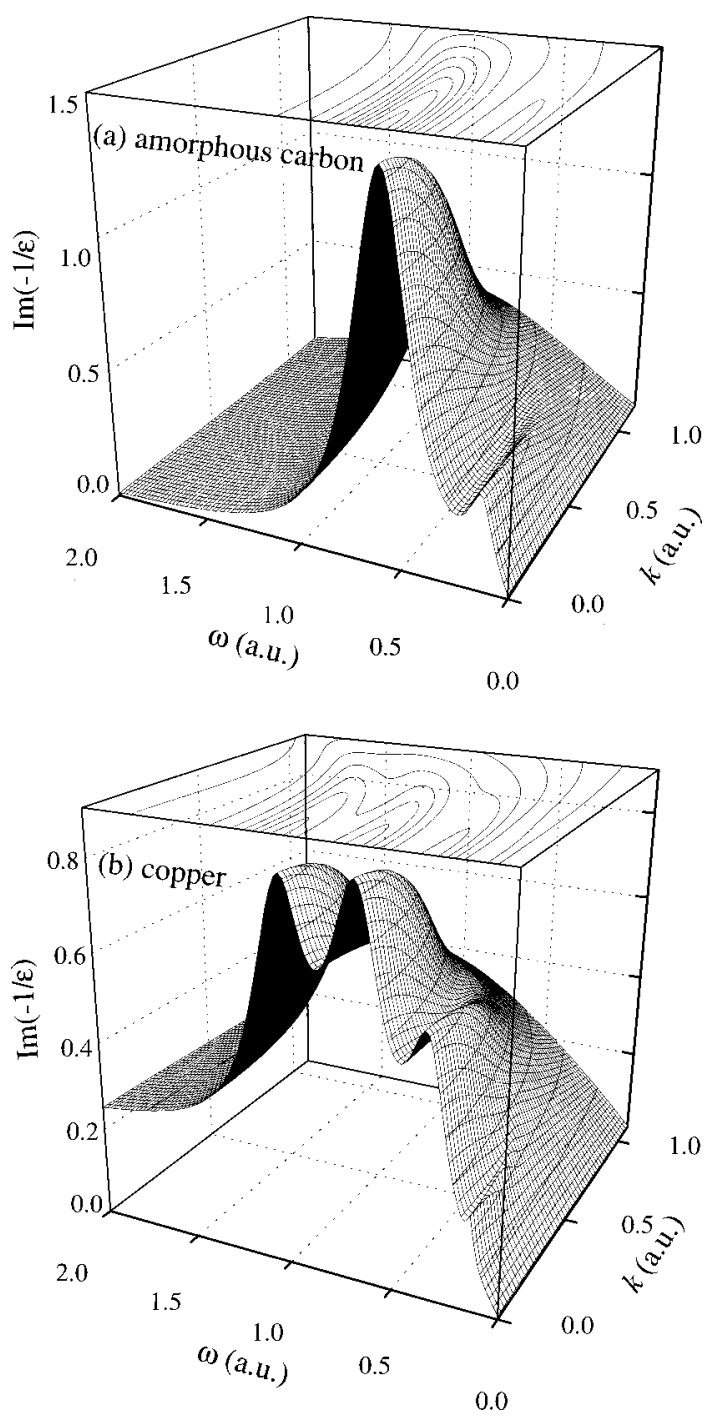

FIG. 3. Tridimensional plot of the Mermin-type ELF as a function of $k$ and $\omega$, for (a) amorphous carbon and (b) copper.

where the vector $\vec{r}$ is measured from the instantaneous position of the proton (so that in this case the time dependence drops out) and $Z$ is the proton charge. In what follows we take $Z=1$.

Due to the axial symmetry around the trajectory of the moving proton we use the cylindrical coordinates $z$ and $\rho$, that represent the parallel and perpendicular projection of the vector $\vec{r}$ relative to the direction of motion. Then the induced potential may be expressed more explicitly as

$$
\phi_{\text {ind }}(z, \rho)=\frac{2}{\pi v} \int_{0}^{\infty} \frac{d k}{k} \int_{0}^{k v} d \omega J_{0}\left(\rho \sqrt{k^{2}-\omega^{2} / v^{2}}\right)\left\{\cos \left(\frac{\omega z}{v}\right) \operatorname{Re}\left[\frac{1}{\epsilon(k, \omega)}-1\right]-\sin \left(\frac{\omega z}{v}\right) \operatorname{Im}\left[\frac{1}{\epsilon(k, \omega)}-1\right]\right\},
$$

where $J_{0}(x)$ is the Bessel function of zero order.

In order to obtain an appropriate expression for $\operatorname{Re}[1 / \epsilon(k, \omega)]$, appearing in the above expression, we use the KramersKronig relation [7]

$$
\operatorname{Re}\left[\frac{1}{\epsilon(k, \omega)}\right]-1=\frac{1}{\pi} \mathcal{P} \int_{-\infty}^{+\infty} d \omega^{\prime} \frac{1}{\omega^{\prime}-\omega} \operatorname{Im}\left[\frac{1}{\epsilon\left(k, \omega^{\prime}\right)}\right],
$$


where $\mathcal{P}$ denotes the principal part of the integral. Taking into account that the energy loss function is given by a linear combination of Mermin-type ELF [see Eq. (6)], we obtain

$$
\operatorname{Re}\left[\frac{1}{\epsilon(k, \omega)}-1\right]=\sum_{i=1}^{N} A_{i} \operatorname{Re}\left[\frac{1}{\epsilon_{\mathrm{M}}\left(k, \omega ; \omega_{i}, \gamma_{i}\right)}-1\right]
$$

In the same way, the induced electric field is given by

$$
\overrightarrow{\mathcal{E}}_{\text {ind }}(\vec{r})=-\vec{\nabla} \phi_{\text {ind }}(\vec{r})=-\frac{1}{2 \pi^{2}} \int \frac{d^{3} k}{k^{2}} i \vec{k} e^{i \vec{k} \cdot \vec{r}}\left[\frac{1}{\epsilon(k, \vec{k} \cdot \vec{v})}-1\right],
$$

which may be broken down into its parallel and perpendicular components with respect to the direction of motion of the proton

$$
\begin{gathered}
\mathcal{E}_{\text {ind }, z}(z, \rho)=\frac{-2}{\pi v^{2}} \int_{0}^{\infty} \frac{d k}{k} \int_{0}^{k v} d \omega \omega J_{0}\left(\rho \sqrt{k^{2}-\omega^{2} / v^{2}}\right)\left\{\sin \left(\frac{\omega z}{v}\right) \operatorname{Re}\left[\frac{1}{\epsilon(k, \omega)}-1\right]+\cos \left(\frac{\omega z}{v}\right) \operatorname{Im}\left[\frac{1}{\epsilon(k, \omega)}-1\right]\right\} \\
\mathcal{E}_{\text {ind, } \rho}(z, \rho)=\frac{-2}{\pi v} \int_{0}^{\infty} \frac{d k}{k} \int_{0}^{k v} d \omega \sqrt{k^{2}-\omega^{2} / v^{2}} J_{1}\left(\rho \sqrt{k^{2}-\omega^{2} / v^{2}}\right)\left\{\cos \left(\frac{\omega z}{v}\right) \operatorname{Re}\left[\frac{1}{\epsilon(k, \omega)}-1\right]-\sin \left(\frac{\omega z}{v}\right) \operatorname{Im}\left[\frac{1}{\epsilon(k, \omega)}-1\right]\right\}
\end{gathered}
$$

where $J_{1}(x)$ is the Bessel function of the first order.

The stopping power (or average energy loss per unit path length) $S_{p} \equiv\langle d E / d x\rangle$ is determined by the retarding force acting on the moving proton, which in this formulation is directly given by the value of the induced electric field at the instantaneous position of the proton, namely,

$$
S_{p}=-\frac{\vec{v}}{v} \overrightarrow{\mathcal{E}}_{\text {ind }}(\vec{r}=\overrightarrow{0}) .
$$

Substituting the parallel component of the electric field, Eq. (15), into Eq. (17) we finally get

$$
S_{p}=\frac{2}{\pi v^{2}} \int_{0}^{\infty} \frac{d k}{k} \int_{0}^{k v} d \omega \omega \operatorname{Im}\left[\frac{-1}{\epsilon(k, \omega)}\right]
$$

In the following we discuss the differences in the induced potentials and electric fields arising from the use of the different dielectric models for each of the elements previously considered.

\section{A. Wake potential}

We consider here the calculation of the induced potential $\phi_{\text {ind }}$ for the materials indicated before. To evaluate $\phi_{\text {ind }}$ according to Eq. (11) we have used Mermin-type ELF with the parameters listed in Tables I and II, and Lindhard-type ELF with the plasmon frequencies given in Table III. These latter values were taken as representative of different experimental data sets.

In Figs. 4(a)-4(b) we show the values of the wake potential calculated along the projectile trail (i.e., at $\rho=0$ ), for a proton moving with velocities $v=1,5$, and 10 a.u. in amorphous carbon and copper. The general shape of the wake potential derived from Eq. (11) shows a damped oscillatory behavior in the longitudinal direction behind the projectile; the pattern of these oscillations decreases exponentially in the transversal direction. Also, this wake potential extends slightly ahead of the projectile.

In the case of aluminum the differences between the results obtained from both ELF models are very small. In the case of silicon the differences between both models are much larger than for aluminum, and these differences increase both with velocity and distance from the moving proton. For amorphous carbon [see Fig. 4(a)], the differences are still much bigger than for aluminum and silicon, and they are important also for points very close to the ion; hence, in this case significant differences are expected also for the stopping powers derived from both models. Finally, we find in the copper target the largests discrepancies between the wake potential predicted by both ELF models [see Fig. 4(b)]; in addition, we notice that the Mermin-type ELF causes the complete disappearance of the oscillatory behavior, usually associated with the wakes produced by swift particles, whereas the wake potential derived from the Lindhard-type ELF always preserves the oscillating character. Thus, a realistic description of the dispersive properties of the real material gives place to a strong damping of the collective oscillations and a relaxation of the spatial distribution.

These differences between the wake potentials predicted from both dielectric models may turn out to be relevant for the analysis of experiments where the effects of the local field or wake potential are explored. As an example of this question we will next consider the values of the stopping force (or stopping power) acting on the moving particle.

\section{B. Stopping power}

We consider now how the stopping power values calculated here compare with experimental data. The proton stop-

TABLE III. Values of the plasmon frequencies of aluminum, silicon, amorphous carbon, and copper used with the Lindhard-type ELF description.

\begin{tabular}{ccccc}
\hline \hline & Aluminum & Silicon & Amorphous carbon & Copper \\
\hline$\omega_{\text {pl }}$ (a.u.) & 0.551 & 0.620 & 0.79 & 0.702 \\
\hline \hline
\end{tabular}



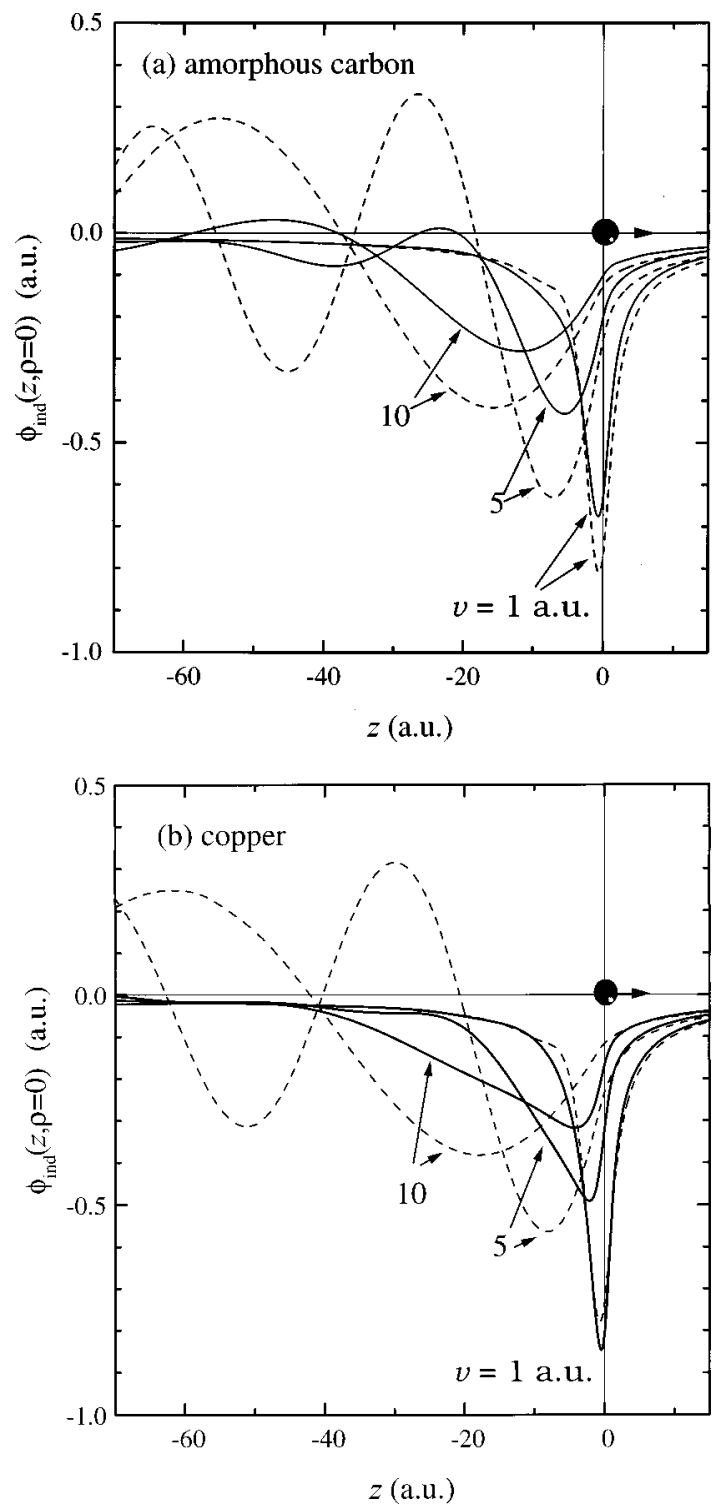

FIG. 4. Wake potential at $\rho=0$ created by a proton that moves in (a) amorphous carbon and (b) copper, for different velocities: $v$ $=1$, 5, and 10 a.u. The solid lines are the results obtained with the Mermin-type ELF, and the dashed lines are derived from the Lindhard ELF.

ping power $S_{p}$ was evaluated from Eq. (18), for the four materials discussed in this work. The energy-loss function, $\operatorname{Im}(-1 / \epsilon)$, was modeled as before, using either a Lindhard or a Mermin representation. In Figs. 5(a)-5(d) the results derived from both procedures are compared with available experimental data in the velocity range $v \leqslant 10$ a.u. It should be noted the wide spread of the experimental data, due to the different ways in which the energy-loss measurements are done [52-55]. The sources of these experimental data are indicated in the corresponding figure.

For the aluminum target, Fig. 5(a), the results of $S_{p}$ derived from both models practically coincide for low velocities, but at higher velocities the use of the Mermin-type ELF improves the results, providing higher stopping powers than with the Lindhard ELF, the former being closer to the experimental data.
For silicon, Fig. 5(b), which is also well described by a single-peaked ELF, a similar behavior of $S_{p}$ is predicted; the results obtained from both ELF models differ mostly near the maximum value of $S_{p}$ and in the high velocity tail, with the Mermin-type ELF providing the better agreement with the experimental data.

The predictions of $S_{p}$ of amorphous carbon, obtained from both ELF models, differ in the values around the maximun, but coincide at low and high velocities, Fig. 5(c). The characterization of amorphous carbon deserves a comment because its density depends markedly on the manner in which it was prepared, but most of the experimental papers do not provide explicitly that value; then, taking into account that many old samples were prepared as arc evaporated carbon (a method that has recently [97] been recognized to produce the allotropic form $\mathrm{C}_{60}$ ), we have considered that the density corresponding to the older works was $1.7 \mathrm{~g} / \mathrm{cm}^{3}$, while that of the more recent ones was $2 \mathrm{~g} / \mathrm{cm}^{3}$ [98]. The stopping power derived from the Mermin-type ELF shows a satisfactory agreement with the experimental data in the full range of velocities we are discussing.

The stronger discrepancies between the $S_{p}$ predictions derived from the Lindhard and the Mermin-type ELF can be seen in Fig. 5(d) for copper, the element with the more complex electronic estructure among those discussed in this work. Again, the stopping power calculated using the Mermin-type ELF agrees very well with the experimental data. Thus, in the cases of carbon and copper the differences in $S_{p}$ when using both models for the ELF are so large as to completely invalidate the descriptions based on the simplest free-electron gas formalism.

It is worth noting that, for the four materials we have considered, the theoretical predictions obtained from the Mermin-type ELF agree fairly well with the experimental data, in the whole range of velocities discussed in this paper.

\section{CONCLUSIONS}

We have presented a more accurate description of the energy-loss function of four elements having different and characteristic electronic properties: aluminum, representing the properties of a good metal; amorphous carbon, characterized by a double-plasma resonance; a typical semiconductor, like silicon; and copper, which shows a rich optical spectrum-due to a complex electronic band structure-as observed in other transition metals [42]. The present model, based on a combination of Mermin-type energy-loss functions, provides a consistent description of these materials that satisfies the $f$-sum rule for all values of wave numbers; the use of analytical functions, with the parameters provided in the Tables I and II may be useful for other calculation purposes.

We have used this representation to get a closer description of the dynamical interactions and excitations induced by swift ions in solids. In particular, we have calculated some of the most relevant quantities for studies of ion-solid interactions, namely, the induced potential and stopping power for swift protons moving in the material.

We have analyzed the main differences derived from this representation as compared with the widely used freeelectron gas description (usually based on Lindhard or plas- 

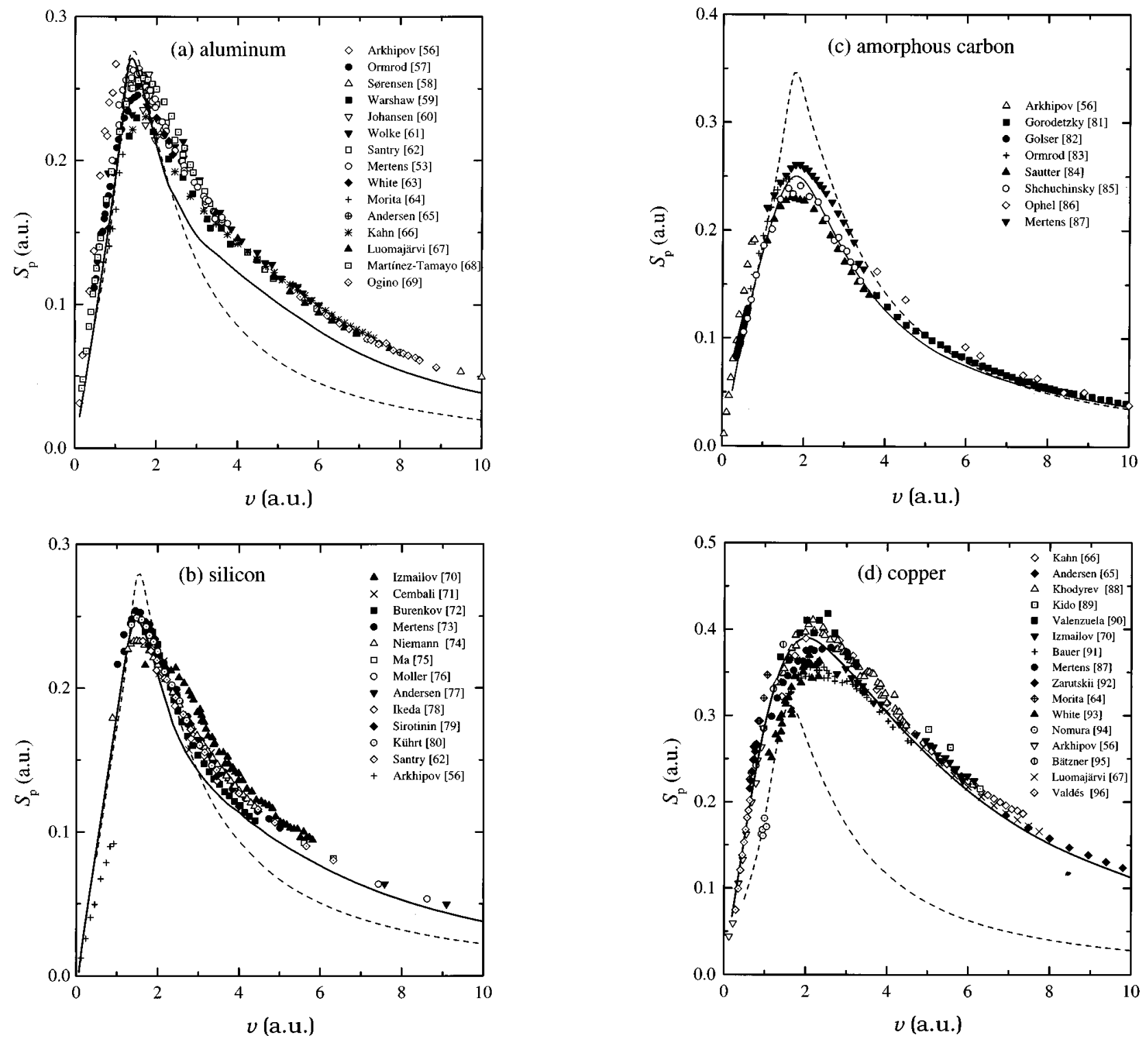

FIG. 5. Stopping power of (a) aluminum, (b) silicon, (c) amorphous carbon, and (d) copper, as a function of the proton velocity $v$. The solid lines correspond to calculations of $S_{p}$ made with the Mermin-type ELF, whereas the dashed lines were obtained using the Lindhard ELF. The sources of the experimental data are indicated in each figure (Refs. [53,56-96]).

mon pole approximations). Of the four elements here studied, we find good agreement between both ELF models for the case of aluminum, an acceptable behavior for silicon, and large differences between these models for the cases of amorphous carbon and copper. The case of silicon shows an interesting behavior, since the results for the wake potential present important discrepancies between both (Lindhard and Mermin) ELF models at large distances, whereas the local values (close to the moving ion) of the potential and electric field, calculated with both models, show a reasonable agreement.

These differences should be relevant in experimental and theoretical investigations of ion-solid interaction processes, where the use of simplified models may lead in some cases to erroneous or misleading results. The magnitude of the differences in the wake potential and stopping powers here obtained illustrates the importance of using models that de- scribe more accurately the optical and dielectric properties of real materials.

The current abundance of data on dielectric properties existing in the literature, over extended ranges of frequencies and for many elements or composites, obtained either from optical data or electron-energy-loss spectroscopies, provide the possibility to extend the present analysis to nearly all materials of experimental interest.

\section{ACKNOWLEDGMENTS}

This work was supported in part by the Spanish Dirección General de Investigación Científica y Técnica (Project Nos. PB95-0689 and PB96-1118). C.D.D. thanks the Instituto de Cooperación Iberoamericana for a grant. N.R.A. wishes to thank the Conselleria d'Educació i Ciència de la Generalitat Valenciana for their support under the program PROPIO. 
[1] E. Fermi, Z. Phys. 29, 315 (1924); Phys. Rev. 56, 1242 (1939); 57, 485 (1940).

[2] D. Pines and D. Bohm, Phys. Rev. 85, 338 (1952); D. Bohm and D. Pines, ibid. 92, 609 (1953).

[3] J. Lindhard, K. Dan. Vidensk. Selsk. Mat. Fys. Medd. 28, 8 (1954).

[4] J. Hubbard, Proc. R. Soc. London, Ser. A 68, 976 (1955).

[5] P. Nozières and D. Pines, Nuovo Cimento 9, 470 (1958).

[6] R. H. Ritchie, Phys. Rev. 114, 644 (1959).

[7] D. Pines, Elementary Excitations in Solids (Benjamin, New York, 1964).

[8] D. Penn, Phys. Rev. 128, 2093 (1962).

[9] W. Brandt and J. Reinheimer, Phys. Rev. B 2, 3104 (1970).

[10] E. Tosatti and G. P. Parravicini, J. Phys. Chem. Solids 32, 623 (1971).

[11] J. P. Walter and M. L. Cohen, Phys. Rev. B 5, 3101 (1972).

[12] M. A. Kumakhov and F. F. Komarov, Energy Loss and Ion Ranges in Solids (Gordon and Breach, New York, 1981).

[13] Interaction of Charged Particles with Solids and Surfaces, Vol. 271 of NATO Advanced Studies Institute Series, Series B: Physics, edited by A. Gras-Martí, H. M. Urbassek, N. R. Arista, and F. Flores (Plenum, New York, 1991).

[14] C. J. Tung, J. C. Ashley, and R. H. Ritchie, Surf. Sci. 81, 427 (1979).

[15] N. D. Mermin, Phys. Rev. B 1, 2362 (1970).

[16] R. H. Ritchie and A. Howie, Philos. Mag. 36, 463 (1977).

[17] J. C. Ashley, J. J. Cowan, R. H. Ritchie, V. E. Anderson, and J. Hoelzl, Thin Solid Films 60, 361 (1979).

[18] J. C. Ashley, J. Phys.: Condens. Matter. 3, 2741 (1991).

[19] I. Abril, R. Garcia-Molina, and N. R. Arista, Nucl. Instrum. Methods Phys. Res. B 90, 72 (1994).

[20] D. J. Planes, R. Garcia-Molina, I. Abril, and N. R. Arista, J. Electron Spectrosc. Relat. Phenom. 82, 23 (1996).

[21] N. Bohr, K. Dan. Vidensk. Selsk. Mat. Fys. Medd. 24, 19 (1948).

[22] J. Neufeld and R. H. Ritchie, Phys. Rev. 98, 1632 (1955).

[23] P. M. Echenique, R. H. Ritchie, and W. Brandt, Phys. Rev. B 20, 2567 (1979).

[24] W. Brandt, A. Ratkowski, and R. H. Ritchie, Phys. Rev. Lett. 33, 1325 (1974).

[25] R. Laubert and F. K. Chen, Phys. Rev. Lett. 40, 174 (1978).

[26] N. R. Arista, Phys. Rev. B 18, 1 (1978).

[27] I. Abril, M. Vicanek, A. Gras-Martí, and N. R. Arista, Nucl. Instrum. Methods Phys. Res. B 67, 56 (1992); M. Vicanek, I. Abril, N. R. Arista, and A. Gras-Martí, Phys. Rev. A 46, 5745 (1992).

[28] F. J. Pérez-Pérez, I. Abril, R. Garcia-Molina, and N. R. Arista, Phys. Rev. A 54, 4145 (1996); F. J. Pérez-Pérez, I. Abril, N. R. Arista, and R. Garcia-Molina, Nucl. Instrum. Methods Phys. Res. B 115, 18 (1996).

[29] C. Denton, F. J. Pérez-Pérez, I. Abril, R. Garcia-Molina, and N. R. Arista, Europhys. Lett. 35, 499 (1996).

[30] Z. Vager, D. S. Gemmell, and B. J. Zabransky, Phys. Rev. A 14, 638 (1976).

[31] J. Remillieux, Nucl. Instrum. Methods 170, 31 (1980).

[32] D. S. Gemmell, Nucl. Instrum. Methods Phys. Res. 194, 255 (1982).

[33] F. Bell, H.-D. Betz, H. Panke, and W. Stehling, J. Phys. B 9, L443 (1976).

[34] J. P. Rozet, A. Chetioui, P. Bouisset, D. Vernhet, A. Touati, C. Stephan, and J. P. Grandin, Phys. Rev. Lett. 58, 337 (1987).
[35] P. Nicolai, M. Chabot, J. P. Rozet, M. F. Politis, A. Chetioui, C. Stephan, A. Touati, D. Vernhet, and K. Wohrer, J. Phys. B 23, 3609 (1990).

[36] P. M. Echenique, F. J. García de Abajo, V. H. Ponce, and M. E. Uranga, Nucl. Instrum. Methods Phys. Res. B 96, 583 (1995).

[37] J. Burgdörfer, Nucl. Instrum. Methods Phys. Res. B 67, 1 (1992).

[38] J. Daniels, C. v. Festenberg, H. Raether, and K. Zeppenfeld, Springer Tracts in Modern Physics Vol. 54, edited by G. Höhler (Springer, Berlin, 1970), p. 77.

[39] F. Wooten, Optical Properties of Solids (Academic, San Diego, 1972).

[40] H.-J. Hagemann, E. Gudat, and C. Kunz, Deutsches Elecktronen-Synchroton Report No. DESY SR-74/7, Hamburg, 1974 (unpublished); J. Opt. Soc. Am. 65, 742 (1975).

[41] R.-P. Haelbich, M. Iwan, and E. E. Koch (Zentralstelle für Atomkernenergie-Dokumentation, Physics Data No. 8-1, Karlsruhe, 1997) (unpublished).

[42] R. Raether, Excitations of Plasmons and Interband Transitions by Electrons, Springer Tracts in Modern Physics Vol. 88 (Springer, Berlin, 1980).

[43] J. H. Weaver, C. Krafka, D. W. Lynch, and E. E. Koch, (Fachinformationszentrum, Physics Data No. 18-1 and 18-2, Karlsruhe, 1981) (unpublished).

[44] Handbook of Optical Constants of Solids. Vols. I and II, edited by E. D. Palik (Academic, Orlando, 1985, 1991).

[45] CRC Handbook of Chemistry and Physics, 73rd ed. edited by D. R. Lide (CRC Press, Boca Raton, 1992).

[46] B. L. Henke, P. Lee, T. J. Tanaka, R. L. Shimabukuro, and B. K. Fujikawa, At. Data Nucl. Data Tables 27, 1 (1982).

[47] J. Cazaux and P. Gramari, J. Phys. (France) 38, L133 (1977).

[48] E. A. Taft and H. R. Philipp, Phys. Rev. 138, A197 (1965).

[49] U. Büchner, Phys. Status Solidi B 81, 227 (1977).

[50] P. E. Batson and J. Silcox, Phys. Rev. B 27, 5224 (1983).

[51] B. S. Yarlagadda, J. E. Robinson, and W. Brandt, Phys. Rev. B 17, 3473 (1978).

[52] D. Semrad, P. Bauer, F. Aumayr, P. Huber, and W. Obermann, Nucl. Instrum. Methods Phys. Res. 218, 811 (1983).

[53] P. Mertens, P. Bauer, and D. Semrad, Nucl. Instrum. Methods Phys. Res. B 15, 91 (1986).

[54] P. Bauer, Nucl. Instrum. Methods Phys. Res. B 27, 301 (1987).

[55] P. Mertens, Nucl. Instrum. Methods Phys. Res. B 27, 315 (1987).

[56] E. P. Arkhipov and Yu. V. Gott, Zh. Éksp. Teor. Fiz. 56, 1146 (1969) [Sov. Phys. JETP 29, 615 (1969)].

[57] J. H. Ormrod, J. R. Macdonald, and H. E. Duckworth, Can. J. Phys. 43, 275 (1965).

[58] H. Sørensen and H. H. Andersen, Phys. Rev. B 8, 1854 (1973).

[59] S. D. Warshaw, Phys. Rev. 76, 1759 (1949).

[60] A. Johansen, S. Steenstrup, and T. Wohlenberg, Radiat. Eff. 8 , 31 (1971).

[61] R. L. Wolke, W. N. Bishop, E. Eichler, N. R. Johnson, and G. D. O'Kelley, Phys. Rev. 129, 2591 (1963).

[62] S. D. Santry and R. D. Werner, Nucl. Instrum. Methods Phys. Res. 188, 211 (1981).

[63] W. White and R. M. Mueller, J. Appl. Phys. 38, 3660 (1967).

[64] K. Morita, H. Akimune, and T. Suita, J. Phys. Soc. Jpn. 25, 1525 (1968).

[65] H. H. Andersen, J. F. Bak, H. Knudsen, and B. R. Nielsen, Phys. Rev. A 16, 1929 (1977). 
[66] D. Kahn, Phys. Rev. 90, 503 (1953).

[67] M. Luomajärvi, Radiat. Eff. 40, 173 (1979).

[68] G. Martínez-Tamayo, J. C. Eckardt, G. H. Lantschner, and N. R. Arista, Phys. Rev. A 54, 3131 (1996).

[69] K. Ogino, T. Kiyosawa, and T. Kiuchi, Nucl. Instrum. Methods Phys. Res. B 33, 155 (1988).

[70] Sh. Z. Izmailov, E. I. Sirotinin, and A. F. Tulinov, Nucl. Instrum. Methods 168, 81 (1980).

[71] F. Cembali and F. Zignani, Radiat. Eff. 31, 169 (1977).

[72] A. F. Burenkov and F. F. Komarov, in Proceedings of the 9th All-Union Conference on the Physics of Charged-Particle Interactions with Single Crystals (Moscow State University Publishers, Moscow, 1978), p. 62.

[73] P. Mertens and P. Bauer, Nucl. Instrum. Methods Phys. Res. B 33, 133 (1988).

[74] D. Niemann, G. Konac, and S. Kalbitzer, Nucl. Instrum. Methods Phys. Res. B 118, 11 (1996).

[75] Z. Ma, J. Liu, and P. Zhu, Chin. Phys. Lett. 7, 226 (1990).

[76] S. P. Moller, Nucl. Instrum. Methods Phys. Res. B 48, 1 (1990).

[77] L. H. Andersen, P. Hvelplund, H. Knudsen, S. P. Moller, J. O. P. Pedersen, E. Uggerhoj, K. Elsener, and E. Morenzoni, Phys. Rev. Lett. 62, 1731 (1989).

[78] A. Ikeda, K. Sumitomo, T. Nishioka, and Y. Kido, Nucl. Instrum. Methods Phys. Res. B 115, 34 (1996).

[79] E. I. Sirotinin, A. F. Tulinov, V. A. Khodyrev, and V. N. Mizgulin, Nucl. Instrum. Methods Phys. Res. B 4, 337 (1984).

[80] E. Kührt, K. Lenkeit, and F. Täubner, Phys. Status Solidi B 66, K131 (1981).

[81] S. Gorodetzky, A. Chevallier, A. Pape, J. Cl. Sens, A. M.
Bergdolt, M. Bres, and R. Armbruster, Nucl. Phys. A 91, 133 (1967).

[82] R. Golser, Ch. Eppacher, and D. Semrad, Nucl. Instrum. Methods Phys. Res. B 67, 69 (1992).

[83] J. H. Ormrod and H. E. Duckworth, Can. J. Phys. 41, 1424 (1963).

[84] C. A. Sautter and E. J. Zimmerman, Phys. Rev. 140, A490 (1965).

[85] J. Shchuchinsky and C. Peterson, Radiat. Eff. 81, 221 (1984).

[86] T. R. Ophel and G. W. Kerr, Nucl. Instrum. Methods 128, 149 (1975).

[87] P. Mertens and Th. Krist, Nucl. Instrum. Methods Phys. Res. 194, 57 (1982).

[88] V. A. Khodyrev, V. N. Mizgulin, E. I. Sirotinin, and A. F. Tulinov, Radiat. Eff. 83, 21 (1984).

[89] Y. Kido and T. Hioki, Phys. Rev. B 27, 2667 (1983).

[90] A. Valenzuela, W. Meckbach, A. J. Kestelman, and J. C. Eckardt, Phys. Rev. B 6, 95 (1972).

[91] P. Bauer, F. Aumayr, D. Semrad, and B. M. U. Scherzer, Nucl. Instrum. Methods Phys. Res. B 1, 1 (1984).

[92] E. M. Zarutskii, Sov. Phys. Solid State 9, 1172 (1967).

[93] W. White and R. M. Mueller, Phys. Rev. 187, 499 (1969).

[94] A. Nomura and S. Kiyono, Jpn. J. Appl. Phys. 15, 1773 (1976).

[95] H. Bätzner, Ann. Phys. (Leipzig) 25, 233 (1936).

[96] J. E. Valdés, J. C. Eckardt, G. H. Lantschner, and N. R. Arista, Phys. Rev. A 49, 1083 (1994).

[97] W. Krätschmer, L. D. Lamb, K. Fostiropoulos, and D. R. Huffman, Nature (London) 347, 354 (1990).

[98] J. O. Stoner, Nucl. Instrum. Methods Phys. Res. A 303, 94 (1991). 